\title{
The successful application of simulation-based training in thoracic surgery residency
}

\author{
Harold M. Burkhart, MD, ${ }^{\mathrm{a}}$ Jeffrey. B. Riley, CCP, ${ }^{\mathrm{b}}$ Sarah. E. Hendrickson, MA, ${ }^{\mathrm{a}}$ George F. Glenn, CCP, \\ James J. Lynch, MD, ${ }^{\mathrm{c}}$ Jackie J. Arnold, RN, ${ }^{\mathrm{d}}$ Joseph A. Dearani, MD, ${ }^{\mathrm{a}}$ Hartzell V. Schaff, MD, ${ }^{\mathrm{a}}$ and \\ Thoralf M. Sundt III, MD
}

Objective: We developed and tested a clinical simulation program in the principles and conduct of cardiopulmonary bypass with the aim of improving confidence and proficiency in this critical aspect of cardiac surgical care.

\begin{abstract}
Methods: Fifteen residents from 6 resident-training programs who reported no prior cardiopulmonary bypass observation or simulation-based perfusion experience participated in a cardiopulmonary bypass course involving both didactic lectures and hands-on simulation. A computer-controlled hydraulic model of the human circulation was used in a specifically designed multidisciplinary simulation center environment to give the participants hands-on training with both basic operations and specific perfusion crisis scenarios. Pretraining and posttraining assessments concerning confidence, knowledge, and applications with regard to cardiopulmonary bypass were administered and compared.
\end{abstract}

Results: Likert scale scores on confidence-related items increased significantly $(P<.001)$, from $59 \% \pm 16 \%$ to $92 \% \pm 8 \%$. Pretraining versus posttraining scores $(72 \% \pm 14 \%)$ on similar cognitive items were not significantly different $(P=.3636)$. Scores on similar open-ended application items before and after training improved from $62 \% \pm 25 \%$ to $85 \pm 10 \%(P<.0001)$. All subjects agreed that simulation-based cardiopulmonary bypass training was superior to classroom- and clinic-based education and that the scenarios enhanced their learning experience.

Conclusions: Simulation-based cardiopulmonary bypass training appears to be an effective technique to build the confidence of thoracic surgery residents regarding knowledge and applications. Scenario-based practice in a specifically designed simulated environment is a valuable adjunct to traditional educational methods and has the potential to improve the training of thoracic residents. (J Thorac Cardiovasc Surg 2010;139:707-12)

Simulation has been used in high-risk industries, such as aviation, for teaching both technical and nontechnical skills. In health care simulation is also rapidly proving itself to be a valuable tool. Experiential learning through simulation has coherence with adult learning principles and is of particular value in training subjects to deal with emergencies without causing harm to patients. Simulation has been shown to be effective in measuring and maintaining trainee skills in laparoscopy, endoscopy, advanced cardiac life support, airway management, and trauma resuscitation. ${ }^{1,2}$

The application of simulation to thoracic surgery has been limited. However, simulation training in cardiothoracic surgery has recently garnered attention as a tool to help attend-

\footnotetext{
From the Division of Cardiovascular Surgery, ${ }^{a}$ the Department of Cardiovascular Perfusion, ${ }^{\mathrm{b}}$ the Department of Anesthesiology, ${ }^{\mathrm{c}}$ and the Mayo Multidisciplinary Simulation Center, ${ }^{\mathrm{d}}$ Mayo Clinic and Foundation, Rochester, Minn.

Disclosures: T. Sundt reports grant support from Sorin.

Read at the Thirty-fifth Annual Meeting of The Western Thoracic Surgical Association, Banff, Alberta, Canada, June 24-27, 2009.

Received for publication June 26, 2009; revisions received Oct 6, 2009; accepted for publication Oct 17, 2009; available ahead of print Dec 28, 2009.

Address for reprints: Harold M. Burkhart, MD, Division of Cardiovascular Surgery,

Mayo Clinic College of Medicine, 200 First St SW, Rochester, MN 55905

(E-mail: burkhart.harold@mayo.edu).

$0022-5223 / \$ 36.00$

Copyright $(c) 2010$ by The American Association for Thoracic Surgery

doi:10.1016/j.jtcvs.2009.10.029
}

ing surgeons educate, as well as improve and rejuvenate, resident learning. ${ }^{3,4}$ Although principles of cardiopulmonary bypass $(\mathrm{CPB})$ can be taught, cardiothoracic surgery residents are rarely formally educated in the operation of the heartlung machine or its full capabilities and limitations. We therefore developed and tested a clinical simulation program in the basic principles, conduct, and interdisciplinary aspects of CPB with the aim of improving residents' confidence and proficiency in this critical aspect of cardiac surgical care.

Our resident curriculum focused on the first 2 levels of Miller's pyramid: knowledge and competence. ${ }^{5}$ We also sought to measure resident confidence and self-efficacy before and after the 2-day program. The multidisciplinary faculty (surgeon, anesthesiologist, and perfusionist) presented the flow and sequence of the application of CPB equipment and techniques, emphasizing the critical communication among the 3 caregivers.

\section{MATERIALS AND METHODS}

Fifteen residents (11 cardiothoracic and 4 cardiac anesthesia residents) who reported no prior $\mathrm{CPB}$ observation or simulation-based perfusion experience participated in a 2-day course involving both didactic lectures and hands-on CPB simulation. Thoracic surgery residents' levels ranged from the first year to the third year of cardiothoracic surgery training. The Mayo Foundation Institutional Review Board approved this study. 


\section{Abbreviations and Acronyms \\ $\mathrm{CPB}=$ cardiopulmonary bypass \\ TSDA $=$ Thoracic Surgery Directors Association}

The program was designed and delivered to build on the Thoracic Surgery Directors Association (TSDA) curricula for resident training (http://www.tsda. org/). A multidisciplinary faculty designed and facilitated the training program. Faculty for the training program included a human-factors professional, anesthesiologists, surgeons, and perfusionists experienced in the application of perfusion-patient simulators. ${ }^{6}$ The program ties the TSDA curricula fundamental CPB concepts to the daily team communication and patient application of extracorporeal circuits, perfusion techniques, and crisis management. The lecture series covered $\mathrm{CPB}$ components, cannulation techniques, anticoagulation and hemostasis, evidence-based perfusion techniques, extracorporeal life support, ventricular assist devices, human factors and team communication, and case scenario analysis for high-risk patients undergoing $\mathrm{CPB}$. Hands-on CPB training in the simulation center included setting up a complete circuit, initiating CPB, cooling, administering cardioplegia, managing complications (eg, arterial air embolism, poor venous drainage, and arrhythmias), and weaning from CPB.

\section{Simulation Center}

The Mayo Clinic Multidisciplinary Simulation Center is a 10,000 square-foot educational facility and state-of-the-art technology center that simulates real patient care settings to complement traditional clinical training for medical professionals. The center has 4 large team training rooms simulating the following environments: emergency department, operating room, intensive care patient room, and endovascular suite. Each room has both a corresponding debriefing and control room. In 2008, the center served more than 6000 learners, including residents, fellows, medical students, nursing staff, and Mayo physician faculty.

The Mayo Clinic Multidisciplinary Simulation Center high-fidelity perfusion simulator uses the Stockert S-5 HLM (Sorin Group USA, Arvada, Colo) and the Orpheus (ULCO Technologies, Marrickville, Australia) computer-controlled hydraulic model of human circulation to simulate CPB for perfusion education and practice. The S-5 is a piece of advance-of-the-art perfusion equipment incorporating numerous safety devices with automatic control of patients' perfusion parameters during emergency conditions. The Orpheus simulator incorporates physiologic, thermodynamic, and pharmacokinetic/pharmacodynamic models and is designed to function as a complete patient substitute for training in the use of HLM and related equipment. The system enables us to deliver simulation-based educational opportunities by presenting specific clinical changes or crises demanding specific interventions (Figure 1).

\section{Statistical Analysis}

Residents were surveyed regarding their prior perfusion education experience at the beginning of the training (Appendix 1) using Likert scale items (4 items; 1 point for "yes" and zero points for "no"; 4 possible points). At the end of the training, using 5 Likert scale items (Appendix 1), residents provided an overall rating for the 2-day course (4 points for "strongly agreed"; 20 possible points). Simple descriptive statistics were provided for the experience survey and the overall course evaluation. At the beginning of the course, residents completed a pretest that included 10 cognitive items (10 points) and 5 cardiopulmonary application (11 points) multiple choice written test items (see Appendix 1 for sample items). At the end of the course, the residents were retested on cognitive (10 possible points) and application (14 possible points) items to measure knowledge acquisition during the course. Session 1 resident scores $(n=9)$ were compared with session 2 resident scores $(n=6)$. Pretraining and posttraining cognitive and ap- plication scores were compared by using the Tukey-Kramer honestly significant difference matched-pairs test for normally distributed data or otherwise the Wilcoxon signed-rank test with JMP 7.0.1 software (SAS Institute, Inc, Chicago, Ill; www.jmp.com).

\section{RESULTS}

All 15 residents completed a 2-day simulation training course. The presurvey and postsurvey results for the 2 training sessions of residents were analyzed. Prior perfusion education scores were very low, with a mean of 0.4 of 4 possible points. Cognitive test scores were $70 \%$ before training and increased to $73 \%$ after training. There was a significant $(P<.001)$ training effect to increase participants' self-reported confidence in their perfusion technology knowledge and application, with scores increasing from a mean of 14.2 to 22.1 of 24 possible points. There was a significant $(P<.0001)$ training effect to increase participants' scores on application test items because of training. Pretraining and posttraining scores went from $62 \%$ to $85 \%$, respectively (Figure 2). Session 2 participants scored significantly higher $(P=.002)$ than session 1 participants on the perfusion application posttest; otherwise, there were no differences between sessions on the other posttraining dimensions.

Participants rated the training very high overall, giving the training a median score of 20 of 20 possible points. All subjects agreed that simulation-based CPB training was superior to classroom- and clinic-based education, that the scenarios met their needs to enhance their learning experience, and that they recommend the course to their peers.

\section{DISCUSSION}

This report represents our initial positive experiences demonstrating the feasibility of using simulation to help train residents in CPB. CPB techniques are explicitly sited in the TSDA comprehensive prerequisite and requisite thoracic surgery residents' educational curriculum (http:// www.tsda.org/). Although cognitive principles of CPB can be taught, cardiovascular surgery and anesthesia residents are rarely formally educated in the operation of the heartlung machine or its full capabilities and limitations. Neither do they learn the perfusionist's perspective. Both are critical to the development of the leadership skills necessary to optimize patient safety and surgical teamwork. $^{7}$ Other issues, such as reduced resident work hours, limit the amount of exposure to real-life CPB crisis scenarios. Simulation offers to fill this gap and could be a valuable adjunct to the training of cardiothoracic surgery residents $\mathrm{s}^{3}$ because it enables participants to gain realworld experience without causing harm to patients as the trend in training moves away from the clinic to controlled simulation environments. ${ }^{8,9}$

Studies have shown that simulation can be used to assess, improve, and maintain trainee skills better than traditional 


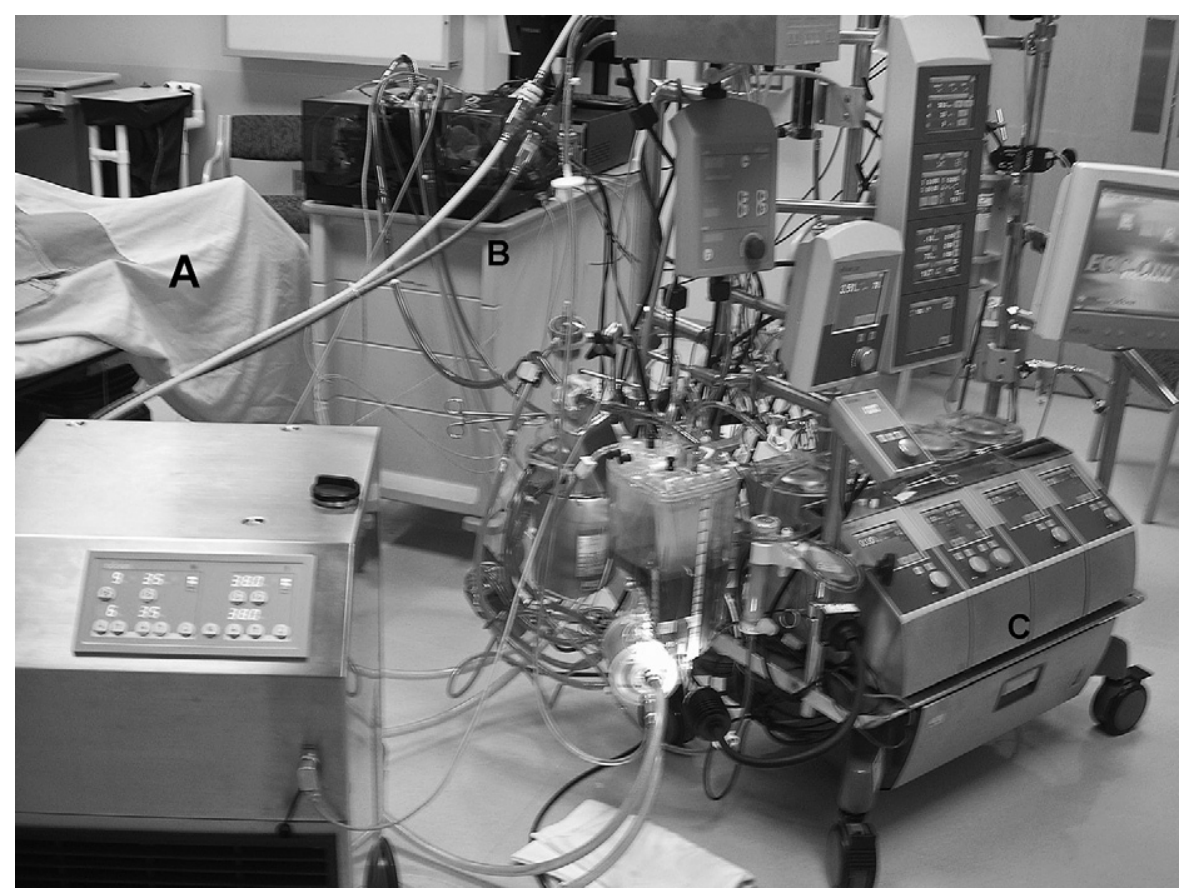

FIGURE 1. Hands-on CPB simulation training set-up includes the patient with anesthesia (A), the Orpheus human circulation model (B), and the Stockert S5 CPB pump (C).

training methods in some disciplines of medicine. ${ }^{10,11}$ Fann and colleagues ${ }^{12}$ recently reported their experience with cardiac surgical simulation in training residents in vascular anastomosis. They showed that after practicing anastomosis with a task station, anastomotic times improved in the majority of residents when assessed with the task station and a beating heart model. Seymour and associates ${ }^{13}$ used virtual reality simulation to train surgical residents in laparoscopic cholecystectomy. They reported improved operating room performance when compared with that of control groups not using simulation. Seymour and associates concluded that the study validated the idea of transfer of simulation-acquired training skills to the operating room. Recent reviews have focused on the purported benefits, as well as shortcomings, of simulation

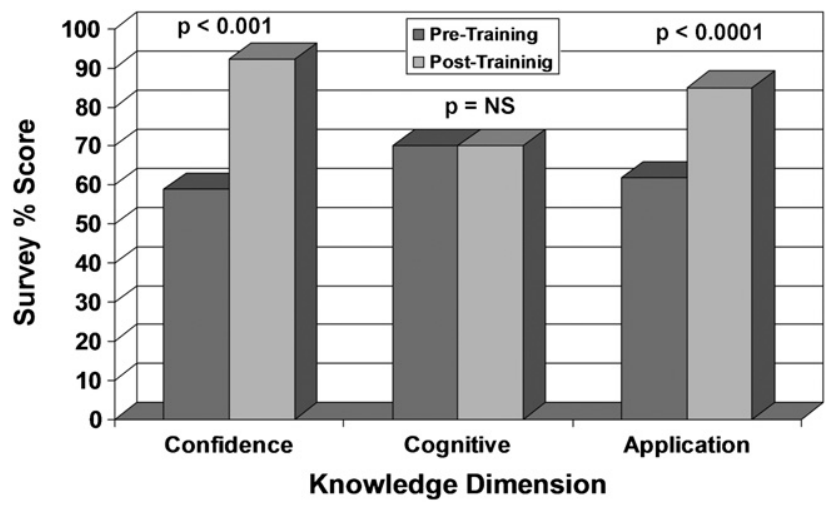

FIGURE 2. Knowledge dimension before and after testing. training in surgery, with most concluding that further study is needed to define the role of simulation. ${ }^{14-16}$

Our aim was to develop a novel simulation-based curriculum for educating residents in the application of knowledge and skills related to the conduct of CPB. This novel curriculum includes exposure to the technical and nontechnical skills of the perfusionist, as well as those of the surgeon and anesthesiologist, thereby providing simulation experience from all 3 points of view. We believe a comprehensive CPB simulation model will provide an invaluable tool for efficient resident training because a wide variety of perfusion challenges can be presented in rapid succession and repeated as necessary to provide optimal didactics. Such a model might also be useful in the future for postgraduate training, as well as postgraduate examination and maintenance of competency.

The use of simulation differs from traditional teaching in the operating room in a number of ways. First, it allows the resident to focus on managing $\mathrm{CPB}$ instead of trying to "fit in" the topic while learning surgical techniques in the operating room from the attending cardiac surgeon. One might argue that the resident could sit at the pump with a perfusionist for a couple of days and would derive similar benefits. The advantage of the simulation course is that it can provide numerous crisis scenarios in a limited amount of time. Following a perfusionist for a few days does not guarantee exposure to these crises and practice with the management. A live patient not being at risk is another added benefit. 
We were hopeful that after completing the CPB simulation course, the residents would demonstrate improvement in their knowledge base, confidence, and application of $\mathrm{CPB}$. The cognitive scores did not improve significantly after the simulation training. In retrospect, this is not surprising and most likely attests to their solid knowledge base with regard to CPB. However, the use of pretraining and posttraining multiple choice surveys to assess resident $\mathrm{CPB}$ knowledge and competence is a limitation to our program. A limitation might have been that the questions were too similar, general, or simple. Future programs will use defined behaviors and more interactive assessments to measure residents' knowledge and application. Another strategy will be to have program directors assess participants' knowledge in the clinic before and after the residents attend the course.

Despite the cognitive scores remaining stable, confidence level and application ability were low in the pretest. However, after 2 days of dedicated didactics and hands-on $\mathrm{CPB}$ simulation, the participants showed significant positive changes in confidence and application knowledge. We would argue that the residents had the cognitive ability, but only after being given the opportunity and instruction in the application of their knowledge did they feel confident in applying that knowledge. Their high satisfaction with the course could serve as confirmation of this assertion. The use of questionnaires before and after training is an accepted qualitative method to assess participant satisfaction. ${ }^{17}$

This report represents our initial experience with using simulation to train residents in CPB. The course is evolving, and we predict that there are several potential avenues that could be helpful not only to the residents but also to the entire surgical team. One possibility is having a simulation set up that involves roles for the surgeon, anesthesiologist, and perfusionist. This would allow the resident to learn not only from the perspective of the surgeon but from the anesthesiologist and perfusionist as well. Another idea would be to combine the CPB simulation with a beating surgical heart model used in the TSDA boot camp for thoracic surgical residents. ${ }^{3}$ This advanced scenario would allow the resident to work on mastering his or her surgical skills (eg, cannulation) while negotiating the institution of CPB. Extending this scenario to include an entire cardiac surgical team could provide an incredible amount of opportunity. Benefits to the surgical team, including reduced practice errors, increased collaboration, and improved mutual respect between the anesthesia, surgical, and perfusion work groups, are just a few that come to mind. ${ }^{7,18}$ We expect that simulation center interactions will also foster the development of more proficient thoracic surgery educators and will help to improve and rejuvenate resident learning related to $\mathrm{CPB} .^{3}$

A limitation to our study was the lack of a formal control group to test and compare with the group using simulation.
We chose to use the pretraining test as the control. We believed this to be acceptable, given that we set out to show simulation would be complementary to traditional cardiac surgery teaching and not necessarily better. Furthermore, documenting improved application in the operating room would strengthen the study. The small numbers of participants is a limitation. However, limiting the course to a small group gave each participant more simulation time during the hands-on portion of the course.

In summary, simulation-based CPB training appears to be a feasible and effective technique to build the confidence of thoracic surgery residents regarding knowledge and applications. Scenario-based practice in a specifically designed simulated environment is a valuable adjunct to traditional education methods and has the potential to improve the training of thoracic residents.

\section{References}

1. Chang L, Petros J, Hess D, Rotondi C, Babineau T. Integrating simulation into a surgical residency program: is voluntary participation effective? Surg Endosc. 2007;21:418-21.

2. Cooke J, Larsen J. Simulation enhances resident confidence in critical care and procedural skills. Fam Med. 2008;40:165-7.

3. Feins R. Expert commentary: cardiothoracic surgical simulation. J Thorac Cardiovasc Surg. 2008;135:485-6.

4. Hicks G Jr, Brown J, Calhoon J, Merrill W. You never know unless you try. J Thorac Cardiovasc Surg. 2008;136:814-5.

5. Miller G. The assessment of clinical skills/competence/performance. Acad Med. 1990;65(suppl):S63-7.

6. Austin J, Riley J. Principles of simulation applied to perfusion technology: 2005 update. J Extra Corpor Technol. 2005;37:118A.

7. Catchpole K, Mishra A, Handa A, McCulloch P. Teamwork and error in the operating room: analysis of skills and roles. Ann Surg. 2008;247:699-706.

8. Carpenter A, Yang S, Uhlig P, Colson Y. Envisioning simulation in the future of thoracic surgical education. J Thorac Cardiovasc Surg. 2008;135:477-84.

9. Park J, MacRae H, Musselman L, Rossos P, Hamstra S, Wolman S, et al. Randomized controlled trial of virtual reality simulator training: transfer to live patients. Am J Surg. 2007;194:205-11.

10. Dunn W, Murphy J. Simulation: about safety, not fantasy [letter]. Chest. 2008; 133:6-8.

11. Wayne D, Didwania A, Feinglass J, Fudale J, Barsuk J, McGaghie W. Simulation-based education improves quality of care during cardiac arrest team responses at an academic teaching hospital: a case-control study. Chest. 2008; 133:56-61.

12. Fann JI, Caffarelli AD, Georgette G, Howard SK, Gaba DM, Youngblood P, et al. Improvement in coronary anastomosis with cardiac surgery simulation. J Thorac Cardiovasc Surg. 2008;136:1486-91.

13. Seymour N, Gallagher A, Roman S, O'Brien M, Bansal V, Andersen D, et al. Virtual reality training improves operating room performance: results of a randomized, double-blinded study. Ann Surg. 2002;236:458-64.

14. Dunkin B, Adrales GL, Apelgren K, Mellinger JD. Surgical simulation: a current review. Surg Endosc. 2007;21:357-66.

15. Sturm L, Windsor J, Cosman P, Cregan P, Hewett P, Maddern G. A systematic review of skills transfer after surgical simulation training. Ann Surg. 2008;248: 166-79.

16. Tavakol M, Mohagheghi M, Dennick R. Assessing the skills of surgical residents using simulation. J Surg Educ. 2008;65:77-83.

17. Tedesco MM, Pak JJ, Harris EJ Jr, Krummel TM, Dalman RL, Lee JT. Simulation-based endovascular skills assessment: the future of credentialing? J Vasc Surg. 2008;47:1008-14.

18. Sundt TM, Henrickson SE, Cima RR. Approaching process improvement from a human factors perspective: seeking leverage from a systems approach. Surgery. 2008;144:96-8. 


\section{Appendix 1. Survey sample items}

Information about your perfusion training experience: short response or check the box

1. I am a(n) $\square$ anesthesia resident $\square$ thoracic surgery resident $\square$ Other.

2. Have you been involved in a perfusion simulation-based education prior to this program? $\square$ yes $\square$ no

3. In the past, have you had the opportunity to "run the pump" or sit with a perfusionist during a procedure in an organized learning situation? $\square$ yes $\square$ no

4. Have you been involved in $\square$ nonhuman or $\square$ human research where you were using the heart-lung machine?

$\square$ yes $\square$ no

Please describe your previous organized perfusion learning experiences:

Your comfort and confidence (affective domain)

\section{Confidence Items}

\section{Pre-}

1. I am confident in my depth of understanding on how the heart lung machine works

2. I am secure in my ability to recognize and identify the main components of the heart lung machine I am adequately prepared for my training program and board exam questions regarding the heart lung machine equipment

3. I am adequately prepared for my training program and board exam questions regarding most perfusion-related techniques

4. Regarding the heart lung machine and perfusion techniques, my training program has adequately prepared me to function as a cardiac surgeon

5. I am comfortable in my ability to supervise and respond to perfusion-related emergency situations

Your perfusion knowledge (cognitive domain) sample items

1. What is the hemostatic defect most commonly observed after CPB?

A. surgical bleeding

B. heparin rebound

C. platelet dysfunction

D. malignant hyperthermia

2. The major cause of an inadequate heparin dose response after heparinization with a normal loading dose is:

A. an AT-III deficiency.

B. an increased protein $\mathrm{C}$ level.

C. a lower than normal circulating blood volume.

D. heparin from a manufacturer's bad production lot.
Post-

1. My activities in the last day and one-half have increased my depth of understanding on how the heart lung machine works

2. I am secure in my ability to recognize and identify the main components of the heart lung machine I believe this course content will help me to be prepared for my training program and board exam questions regarding the heart lung machine equipment

3. Having taken this course has adequately prepared me for my training program and board exam questions regarding most perfusion-related techniques

4. Regarding the heart lung machine and perfusion techniques, this course has adequately prepared me to function as a cardiac surgeon or cardiac anesthesiologist

5. Having completed this course, I am more comfortable in my ability to supervise and respond to perfusion-related emergency situations in the OR

3. The process of temperature correction is a process that corrects for changes that blood gases experience when temperature is changed during the sampling process.
A. mathematical, physical
B. chemical, physiologic
C. logical, metaphysical
D. theoretical, hypothetical

Your application of $\mathrm{CPB} /$ perfusion skills (application domain) sample items

1. List 3 major perfusion incidents or accidents that a thoracic surgeon or anesthesiologist should be able to negotiate. 
1.

2.

3.

2. List 3 safety devices typically mounted on heart-lung machines.

1.

2.

3.
3. Describe your cardioplegic solution, including the final $\left[\mathrm{K}^{\dagger}\right]$ and hematocrit levels injected into the aorta.

$[\mathrm{K}+]=\ldots \mathrm{mM} / \mathrm{L} \mathrm{HCT}=\ldots$

4. What verbal order does the surgeon give to the perfusionist before applying the aortic crossclamp and why?

Why?

Overall course review

Please check the box for the level of agreement that represents your Strongly Agree understanding and perception

1 Simulation-based education techniques are superior to classroom-lecture and clinic-based education techniques.

2 The use of hands-on techniques enhanced my learning experience.

3 The choice of perfusion equipment and scenarios met my educational needs.

4 Overall, I believe the course met my needs regarding orientation to the heart-lung machine and CPB.

5 I would highly recommend this course to my peers. 